\title{
The Anatomy of a Clinical Trial
}

\author{
The West of Scotland Coronary Prevention Study
}

\author{
J. Shepherd A. Gaw \\ on behalf of The West of Scotland Coronary Prevention Study Group
}

Institute of Biochemistry, Royal Infirmary, Glasgow, UK

\section{Key Words}

HMG CoA reductase inhibitor - Pravastatin .

Coronary heart disease prevention - Drug compliance.

Ancillary actions of statins

\begin{abstract}
Objective: Treatment of hyperlipidemia to reduce the risk of ischemic heart disease was, prior to the statin era, disappointingly limited in its ability to yield the benefits expected from the strong relationship known to exist between serum cholesterol and coronary death. Three primary prevention trials, using clofibrate, cholestyramine and gemfibrozil, had achieved modest reductions in fatal and nonfatal coronary events but none was able to extend life overall or even to reduce cardiovascular mortality; and combined analyses of the three raised disquiet over potential links between cholesterol reduction, cancer and aggressive or violent behaviour. The time was therefore ripe to determine whether statins could help prevent that first and all important myocardial infarction. Methods: The West of Scotland Coronary Prevention Study recruited 6,595 45- to 64-year-old men with no history of prior myocardial infarction and with low-density lipoprotein cholesterol in the range 4$6 \mathrm{mmol} / \mathrm{l}$ (155-232 mg/dl). Subjects who had undergone coronary revascularisation or had been hospitalised for angina pectoris in the previous 12 months were excluded, as were those with significant electrocardiographic abnormalities. Participants were randomised to receive pravastatin $40 \mathrm{mg} /$ day or matching placebo and were followed for an average of 4.9 years. Results: Treat-
\end{abstract}

ment with pravastatin reduced the combined risk of fatal and nonfatal myocardial infarction by $31 \%$. Cardiovascular death overall fell by $32 \%$ and the need for coronary revascularisation procedures was reduced by $37 \%$. All of these endpoint benefits were statistically significant. Because there was no increase in non-cardiovascular mortality, the reduction in death from any cause also proved to be statistically significant ( $p=0.051$ by log rank test and $p=0.037$ after adjustment for baseline risk factors). Conclusions: The West of Scotland Coronary Prevention Study is the first to show that cholesterol reduction with pravastatin helps avoid the first myocardial infarction, reduces coronary mortality and extends life. Ongoing exploration of the study database continues to unearth additional surprisingly beneficial effects of the treatment and permits authoritative decision-making on the effective use of lipid-lowering drugs.

Copyright $\odot 2002$ S. Karger AG, Basel

\section{Introduction}

Atheroma was recognised in ancient Egypt, but only recently has its impact on population mortality been fully appreciated. In industrialised countries, coronary atherosclerosis is responsible for the majority of deaths among all adults aged 40 years and over. Although the lesion is multifactorial in origin, its frequent enrichment in cholesterol has led to the growing conviction that it results from a steady and progressive lifetime uptake of plasma cholesterol into the arterial subintimal space. This concept, which accords with epidemiological evidence associating

\begin{tabular}{ll}
\hline KARGER & ( ) 2002 S. Karger AG, Basel \\
Fax +4161306 1234 $3411-7571 / 02 / 0116-0017 \$ 18.50 / 0$ \\
$\begin{array}{l}\text { E-Mail karger@karger.ch } \\
\text { www.karger.com }\end{array}$ & $\begin{array}{l}\text { Accessible online at: } \\
\text { www.karger.com/mpp }\end{array}$
\end{tabular}

Prof. J. Shepherd

Institute of Biochemistry, Royal Infirmary

Glasgow G4 0SF (UK)

Tel. +440 01412114628, Fax +4401415531703

E-Mail james.shepherd@clinmed.gla.ac.uk 
raised circulating cholesterol levels with increased risk of coronary mortality, has led to the evolution of the cholesterol hypothesis which predicts that since hypercholesterolemia is associated with coronary heart disease (CHD), cholesterol-lowering strategies should be instrumental in its avoidance. The West of Scotland Coronary Prevention Study (WOSCOPS) examined that hypothesis [1].

\section{Background}

The results of three major primary prevention trials designed to test the potential of lipid-lowering agents for preventing CHD appeared in the literature prior to WOSCOPS. The first, the World Health Organization (WHO) clofibrate trial [2], demonstrated a reduction in the rate of nonfatal myocardial infarction in the drug-treated group but also indicated that clofibrate therapy was associated with a rise in total mortality. This finding raised doubts over the benefits of widespread use of lipid-lowering agents. The publication of the Lipid Research Clinic's Coronary Primary Prevention Trial (LRC-CPPT) in 1984 [3, 4] and the Helsinki Heart Study (HHS) in 1987 [5] reversed this attitude to some extent, but many still remained skeptical [6-8] because of the inability of these later trials to show a significant effect on coronary or total mortality. In the LRC-CPPT, an 11\% decrease in the level of low density lipoprotein (LDL) cholesterol was associated with a significant decrease of $19 \%$ in cardiac events (fatal plus nonfatal myocardial infarction). The benefits from this study were less than predicted due to compliance problems. In HHS, gemfibrozil was less potent but was palatable and compliance among subjects at trial visits was high. The drug reduced LDL cholesterol and triglyceride levels by 8 and 35\%, respectively, and increased levels of high-density lipoprotein (HDL) cholesterol by about $10 \%$. These lipid changes were associated with a significant reduction (34\%) in the incidence of CHD as measured by the combined fatal plus nonfatal myocardial infarction endpoint.

Neither the LRC-CPPT nor the HHS had the statistical power to address the question of the benefits of lipidlowering agents in preventing coronary death. To do this, it was necessary to: (a) study a population with a higher event rate; (b) increase the sample size, and (c) use a more effective lipid-lowering agent. The WOSCOPS using pravastatin addressed each of these issues. Pravastatin, one of the newly developed 3-hydroxy-3-methylglutaryl coenzyme A (HMG-CoA) reductase inhibitors, is a powerful cholesterol-reducing agent that is easily tolerated

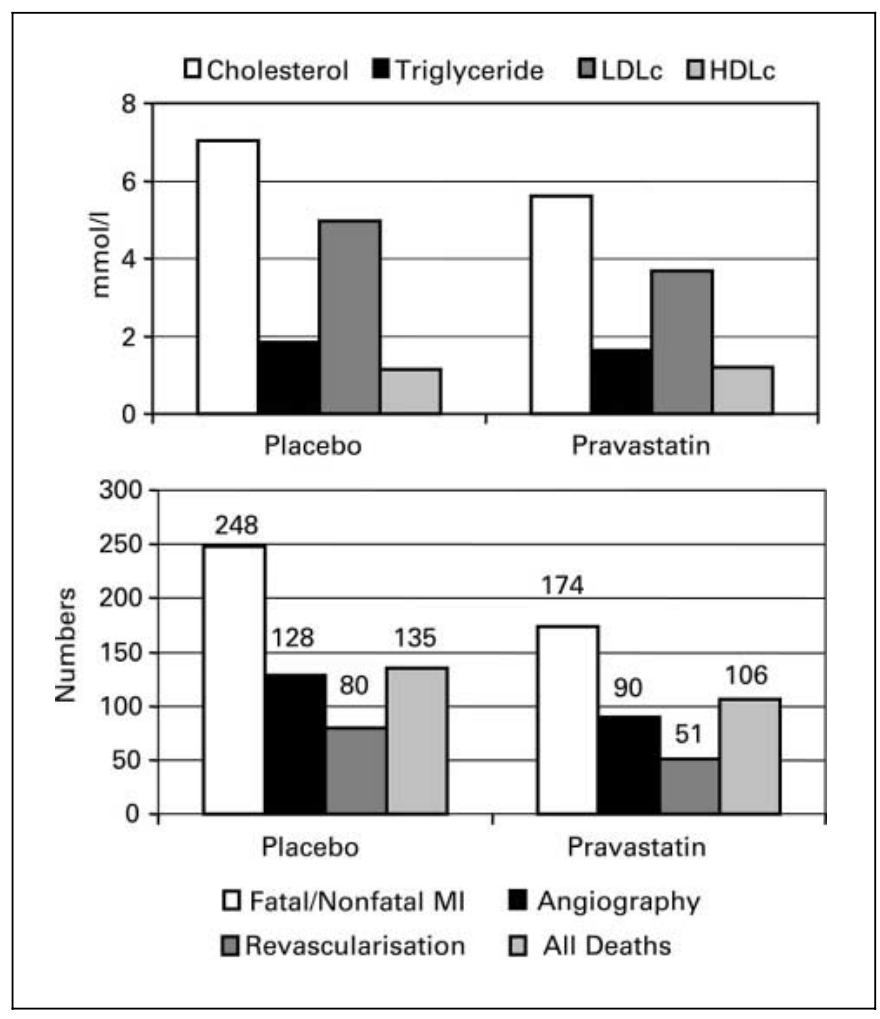

Fig. 1. Primary findings from the West of Scotland Coronary Prevention Study. Pravastatin lowered plasma cholesterol by $20 \%$, triglyceride by $12 \%$, LDL cholesterol by $26 \%$ and raised HDL cholesterol by $5 \%(\mathrm{p}<0.001$ in each case). As a result, fatal and nonfatal MI combined fell and the need for angiography fell $31 \%(\mathrm{p}<0.001$ and $<0.007$, respectively). Revascularisation procedures were reduced $37 \%(\mathrm{p}<0.009)$ and all cause mortality fell $22 \%(\mathrm{p}<0.051)$.

and uniformly effective. The increase in sample size and the recruitment of older men in a high-risk area (Scotland in 1988 had the world's highest incidence of coronary artery disease (CAD) mortality) with associated higher event rates strengthened the power of the study to assess the impact of an improved lipid profile on CAD events.

\section{WOSCOPS Design and Findings}

The West of Scotland Coronary Prevention Study was, in its day, the largest statin-based cholsterol-lowering trial ever conducted in subjects without established coronary heart disease and the first of its kind to use a member of the 3-hydroxy-3-methylglutaryl coenzyme A reductase inhibitor or statin class of lipid-lowering drugs in this population. A group of 6,595 men aged 45-64 years was recruited in the West of Scotland on the basis of a plasma 
Table 1. Benefits of good compliance in WOSCOPS

\begin{tabular}{llll}
\hline Endpoint & \multicolumn{2}{l}{ Percent risk reduction in } \\
\cline { 2 - 4 } & entire cohort & $\begin{array}{l}>75 \% \text { compliers* } \\
\text { (unadjusted) }\end{array}$ & $\begin{array}{l}>75 \% \text { compliers** } \\
\text { (adjusted) }\end{array}$ \\
\hline Definite CHD death or nonfatal MI & $31(\mathrm{p}<0.001)$ & $37(\mathrm{p}<0.001)$ & $38(\mathrm{p}<0.001)$ \\
Cardiovascular death & $32(\mathrm{p}<0.033)$ & $35(\mathrm{p}<0.035)$ & $37(\mathrm{p}<0.029)$ \\
All deaths & $22(\mathrm{p}<0.051)$ & $32(\mathrm{p}<0.017)$ & $32(\mathrm{p}<0.015)$ \\
Revascularisation procedures (CABG and PTCA) & $37(\mathrm{p}<0.009)$ & $46(\mathrm{p}<0.0026)$ & $46(\mathrm{p}<0.031)$ \\
Incident cancers & $-8(\mathrm{NS})$ & $4(\mathrm{NS})$ & $6(\mathrm{NS})$ \\
\hline
\end{tabular}

* log-rank test. ${ }^{* *}$ Cox model.

cholesterol of $6.5-7.8 \mathrm{mmol} / \mathrm{l}(250-300 \mathrm{mg} / \mathrm{dl})$ and an LDL cholesterol between 4.0 and $6.0 \mathrm{mmol} / 1$ (155$232 \mathrm{mg} / \mathrm{dl}$ ). None of the subjects had had a previous myocardial infarction or coronary revascularisation surgery although $5 \%$ had evidence of angina pectoris as determined by a positive self-reported Rose questionnaire [9] and individuals were admitted into the trial with minor ECG abnormalities, the latter being defined as Minnesota Codes 4-2, 4-3 or 5-2, 5-3. This effectively is equivalent to ST depression $\leq 1 \mathrm{~mm}$ with a horizontal or downward sloping ST segment, or $\mathrm{T}$ wave inversion $<5 \mathrm{~mm}$. It should be noted, however, that in WOSCOPS which was a primary prevention study, individuals with more significant ST-T changes were excluded from the Study. The men were randomly assigned to treatment with pravastatin $(40 \mathrm{mg} /$ day $)$ or to a matching placebo. Smoking and cholesterol-lowering dietary advice was provided throughout the study which lasted, on average, 4.9 years. Treatment with pravastatin lowered plasma cholesterol by $20 \%$, LDL cholesterol by $26 \%$, and triglyceride by $12 \%$. HDL cholesterol rose $5 \%$. These lipid changes resulted (fig. 1) in a one-third reduction in fatal and nonfatal myocardial infarctions (174 on pravastatin vs. 248 on placebo). Cardiovascular deaths fell by the same percentage (50 deaths on pravastatin, 73 on placebo), and, since there was no increase in noncardiovascular mortality as a result of pravastatin therapy, deaths from any cause were reduced by about a fifth $(105$ deaths in the pravastatin group vs. 135 on placebo $(\mathrm{p}=0.051$ by the log-rank test and $\mathrm{p}=0.037$ using the Cox model). Unexpectedly, the benefits of therapy began to emerge within about 6 months of initiating therapy and were maintained as long as it continued.

Poor compliance is always a problem in the conduct of primary prevention trials. Patients who do not have overt symptoms of the conditions for which they are being treated are less well motivated to comply with long-term therapy and may be less willing to tolerate minor side effects which could be associated with medication. There is a greater tendency for adverse events which develop after the initiation of therapy to be linked, rightly or wrongly, with the medication being taken. Hence, although it is appropriate that the primary analysis of any randomized trial is based on the intention-to-treat principle, some form of on-treatment analysis is of great interest in the context of primary prevention both to provide a true picture of the benefits of therapy in subjects who are good compliers and to motivate future patients to optimize their compliance. At the same time, it should be acknowledged that analyses, which adjust for on-treatment measures such as compliance, are no longer based on truly randomized comparisons and hence should be interpreted cautiously.

The level of non-compliance in the West of Scotland Study, with approximately $30 \%$ of subjects withdrawn from randomized therapy at 5 years, was similar to that of previous primary prevention trials of cholesterol-lowering drugs [3-5]. This was, in part, due to the causes outlined above and was considerably exacerbated by the cholesterol controversy [6-8] which received a high media profile throughout the study, providing a very difficult climate within which to conduct a trial of this nature. There was no evidence in the trial of any treatment-related pattern of withdrawal from medication. The drug was very well tolerated. Post hoc analysis of the benefits to compliant subjects [10] indicated that they gained more than did the entire cohort, analysed on an intention-to-treat basis (table 1). While subject to the usual subgroup analysis caveats, these findings support the view that physicians ought to take steps to improve patient motivation to adhere to 
prescription advice. The data also carry an important message with regard to assessment of the cost-effectiveness of treatment (see later).

The primary findings of the West of Scotland Study established the safety and efficacy of pravastatin and opened new perspectives on the selection and management of hypercholesterolemic patients at risk of a first myocardial infarction. Paramount in the mind of the practising clinician are the following issues:

(A) Who should be treated?

(B) What is the treatment strategy?

(C) How much will it cost?

The WOSCOPS findings have helped answer these questions.

\section{Lessons from WOSCOPS ${ }^{1}$}

\section{A Who Should Be Treated?}

Interpretation of the results of the West of Scotland Study in its broadest sense would lead to the conclusion that since cholesterol reduction with pravastatin is virtually devoid of side effects all patients eligible for recruitment to the trial would benefit from intervention and consequently merit treatment. However, implementation of such a liberal strategy is clearly economically impractical. Instead, it is more rational to identify and intervene only in those individuals whose risk of an event exceeds an accepted specified value. In 1998, a joint commission from a number of European and other societies with an interest in CHD prevention ruled [11] that a $20 \%$ risk of a vascular event over the next 10 years (or, broadly, a $2 \%$ risk per annum) warranted consideration for intervention, fully aware that such an arbitrary decision required enough flexibility in its interpretation to meet the circumstances of populations with differing financial and healthcare constraints. With that caveat in mind, how can one target individuals for treatment on the basis of their prospective risk of an event? Consideration of the frequency and distribution of cardiovascular events in the placebo group of the WOSCOPS cohort permits such a selection to be made. A number of categorical and continuous coronary heart disease risk predictors were identified

Following publication of the WOSCOPS results the US Federal Drug Administration, on the strength of the study safety data, eliminated the need for repeated liver function testing for pravastatin and recommended only that testing was required prior to and 12 weeks following initiation of effective treatment. and the impact of each risk predictor quantified both individually and in aggregate [12]. As is evident from table 2, age is an important univariate predictor of risk of fatal or nonfatal myocardial infarction as is systolic blood pressure and plasma HDL cholesterol and triglyceride. Total plasma cholesterol or even LDL cholesterol, on the other hand, are much less predictive of the likelihood of disease. Categorical predictors based on preexisting disease (like diabetes, angina pectoris or prescribed nitrate therapy), an unhealthy lifestyle (e.g. cigarette smoking), or psychosocial factors (e.g. widowhood, unemployment or stultified education) again weigh heavily on risk of CHD in their own right (table 2). In aggregate, they permit the constitution of a risk-scoring system (table 3 ) which locates only $9 \%$ of the fatal and nonfatal coronary events in the lowest risk quartile of the placebo group and 45\% in the highest quartile. Similarly, only $5 \%$ of coronary revascularisations and $9 \%$ of deaths from any cause occurred in the lowest risk quartile in distinction to 54 and $56 \%$, respectively, in the highest quartile. Figure 2 translates these arbitrarily designated risk scores into the terms of the European Society Joint Guidelines [11]. Individuals with a 5-year risk of a fatal or nonfatal myocardial infarction of $10 \%$ expressed an arbitrary risk score of 6.2 . Fortyfive percent of all coronary events occurred in individuals with a score above this value and these events were confined to individuals in the highest quartile of risk in the placebo group. In other words, adoption of the European Joint Guidelines will focus on that quarter of hypercholesterolemic coronary event free individuals at highest risk, will successfully identify $45 \%$ of patients in this category who will eventually have a myocardial infarction, and, by inference, will disregard $55 \%$ of all first myocardial infarct victims. Such patients can be located on the basis of their non-lipid risk factor profile, cogent argument that cholesterol screening of asymptomatic individuals should be limited to middle-aged men at high short-term risk of a coronary event, with treatment restricted to this group and to those who present with atherosclerotic disease. Compared with a strategy of untargetted screening and treatment, this approach would ensure the maximum absolute benefit and cost effectiveness of therapy. Treatment of subjects in the top quartile of risk distribution in WOSCOPS would address $45 \%$ of all coronary events and $64 \%$ of coronary fatalities. However, the majority of events $(55 \%)$, which occur in the lower three quartiles of the risk distribution would be missed, even though it is clear that pravastatin induced the same relative reduction in coronary risk across the entire spectrum of the WOSCOPS cohort. But, the number needed to be treated to 
Table 2. Predictors of risk in the WOSCOPS placebo group

\begin{tabular}{llll}
\hline Variate & & \multicolumn{2}{l}{ Definite CHD death or nonfatal MI } \\
\cline { 3 - 4 } & & $\begin{array}{l}\text { univariate risk ratio } \\
(95 \% \mathrm{CI})\end{array}$ & $\begin{array}{l}\text { multivariate risk ratio } \\
(95 \% \mathrm{CI})\end{array}$ \\
\hline a Continuous & Median value & & \\
Age $(5$ years $)$ & 55.2 & $1.35(1.23,1.48)$ & $1.35(1.23,1.48)$ \\
Systolic BP $(20 \mathrm{~mm} \mathrm{Hg})$ & 134 & $1.29(1.17,1.44)$ & \\
Diastolic BP $(10 \mathrm{~mm} \mathrm{Hg})$ & 84 & $1.17(1.07,1.28)$ & $1.19(1.09,1.31)$ \\
Total cholesterol $(20 \mathrm{mg} / \mathrm{dl})$ & 269 & $1.04(0.96,1.13)$ & \\
LDL cholesterol $(20 \mathrm{mg} / \mathrm{dl})$ & 189 & $1.09(0.98,1.21)$ & \\
HDL cholesterol $(10 \mathrm{mg} / \mathrm{dl})$ & 43 & $0.73(0.65,0.82)$ & \\
Log triglyceride $(0.5 \mathrm{log}[\mathrm{mg} / \mathrm{dl}])$ & 5.00 & $1.24(1.10,1.40)$ & \\
Total/HDL cholesterol ratio $(0.5)$ & 6.28 & $1.10(1.07,1.14)$ & $1.08(1.05,1.12)$ \\
\hline Categorical & Prevalence, $\%$ & & \\
Current smoker & 44 & $1.78(1.47,2.16)$ & $1.82(1.49,2.21)$ \\
Diabetes mellitus & 1.2 & $2.63(1.48,4.67)$ & $2.10(1.18,3.73)$ \\
Nitrate consumption & 2.1 & $3.56(2.43,5.23)$ & $1.90(1.26,3.02)$ \\
Angina pectoris & 5.1 & $2.41(1.78,3.26)$ & $1.54(1.07,2.22)$ \\
Family history of CHD & 5.7 & $1.58(1.12,2.22)$ & $1.71(1.22,2.42)$ \\
Widowhood & 2.5 & $2.35(1.54,3.57)$ & $1.66(1.09,2.54)$ \\
Unemployment & 29 & $1.62(1.33,1.96)$ & \\
No school certificate & 56 & $1.26(1.03,1.56)$ & \\
\hline & & &
\end{tabular}

Table 3. CHD risk distribution in the WOSCOPS placebo group

\begin{tabular}{|c|c|c|c|c|}
\hline \multirow[t]{2}{*}{ Endpoint } & \multicolumn{4}{|c|}{ Quartiles of risk score } \\
\hline & 1 & 2 & 3 & 4 \\
\hline \multicolumn{5}{|l|}{ Definite CHD death or nonfatal MI } \\
\hline 5-year probability of event, $\%$ & $<4.48$ & $\geq 4.48,<6.53$ & $\geq 6.53,<9.62$ & $\geq 9.62$ \\
\hline Observed events, $\%$ of total & 9 & 19 & 27 & 45 \\
\hline \multicolumn{5}{|c|}{ Definite or suspect CHD death or nonfatal MI } \\
\hline 5-year probability of event, $\%$ & $<5.27$ & $\geq 5.27,<7.68$ & $\geq 7.68,<11.3$ & $\geq 11.3$ \\
\hline Observed events, $\%$ of total & 9 & 18 & 29 & 44 \\
\hline \multicolumn{5}{|c|}{ Coronary revascularisation (PTCA or CABG) } \\
\hline 5-year probability of event, $\%$ & $<1.27$ & $\geq 1.27,<1.79$ & $\geq 1.79,<2.73$ & $\geq 2.73$ \\
\hline Observed events, $\%$ of total & 5 & 15 & 26 & 54 \\
\hline \multicolumn{5}{|l|}{ Definite or suspect CHD death } \\
\hline 5-year probability of event, $\%$ & $<0.763$ & $\geq 0.763,<1.27$ & $\geq 1.27,<2.20$ & $\geq 2.20$ \\
\hline Observed events, $\%$ of total & 11 & 13 & 12 & 64 \\
\hline \multicolumn{5}{|l|}{ Cardiovascular mortality } \\
\hline 5-year probability of event, $\%$ & $<0.960$ & $\geq 0.960,<1.58$ & $\geq 1.58,<2.71$ & $\geq 2.71$ \\
\hline Observed events, $\%$ of total & 11 & 13 & 17 & 59 \\
\hline \multicolumn{5}{|l|}{ All cause mortality } \\
\hline 5-year probability of event, $\%$ & $<1.82$ & $\geq 1.82,<3.05$ & $\geq 3.05,<5.21$ & $\geq 5.21$ \\
\hline Observed events, $\%$ of total & 9 & 16 & 19 & 56 \\
\hline
\end{tabular}


Fig. 2. Risks and events in the WOSCOPS placebo group. This figure describes the risk of coronary heart disease death or nonfatal myocardial infarction in the WOSCOPS placebo group. The 5 -year risk of an event (right vertical axis) is plotted as a continuous line against their risk score, calculated from their baseline risk factor profile. The cumulative percentage of randomised subjects (left vertical axis) who did (dotted line) or did not suffer an event (dashed line) throughout the trial is also indicated in relation to their risk score. Horizontal line A, representing a 5year risk of $10 \%$, the value targetted by the joint EAS, ESC, ESH guidelines [11], is associated with a risk score of 6.2 or greater, as depicted by the vertical line B. Horizontal lines $C$ and $D$ indicate that $45 \%$ of all coronary events occurred in subjects with a risk score of greater than $6.2(\mathrm{C})$ and that $25 \%$ of the population had a risk score which exceeded this value (D).

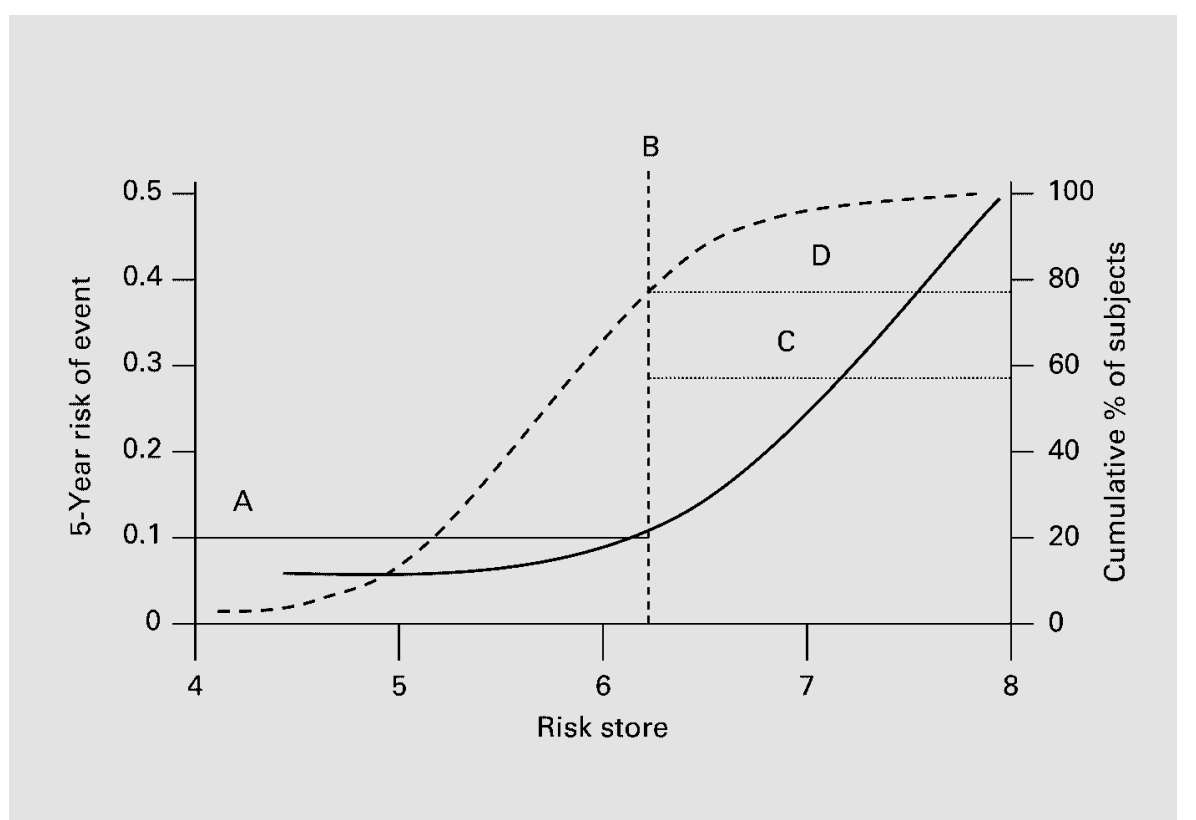

Table 4. Benefits of treatment in WOSCOPS, $4 \mathrm{~S}$ and the MRC Hypertension Trial

\begin{tabular}{|c|c|c|c|c|c|}
\hline \multirow[t]{2}{*}{ Trial (event avoided) } & \multicolumn{2}{|c|}{$\begin{array}{l}\text { Risk of event at } \\
5 \text { years, } \%\end{array}$} & \multirow{2}{*}{$\begin{array}{l}\text { Relative } \\
\text { risk } \\
\text { reduction } \\
\%\end{array}$} & \multirow{2}{*}{$\begin{array}{l}\text { Absolute } \\
\text { risk } \\
\text { reduction } \\
\%\end{array}$} & \multirow{2}{*}{$\begin{array}{l}\text { Number } \\
\text { treated to } \\
\text { avoid one } \\
\text { event }\end{array}$} \\
\hline & placebo & active & & & \\
\hline $\begin{array}{l}\text { WOSCOPS men, } 45-64 \text { years } \\
\text { (definite + suspect CHD death or nonfatal MI) }\end{array}$ & 9.3 & 6.8 & 29 & 2.5 & 40 \\
\hline $\begin{array}{l}\text { 4S men and women, } 35-70 \text { years } \\
\quad \text { (definite + suspect CHD death or nonfatal MI) }\end{array}$ & 25.9 & 18.0 & 34 & 7.9 & 13 \\
\hline $\begin{array}{l}\text { WOSCOPS men, 55-64 years } \\
\text { (definite CHD death or nonfatal MI) }\end{array}$ & 9.8 & 7.3 & 26 & 2.5 & 40 \\
\hline $\begin{array}{l}\text { MRC men, } 55-64 \text { years } \\
\quad \text { (definite fatal or nonfatal stroke) }\end{array}$ & 2.6 & 1.5 & 42 & 1.1 & 91 \\
\hline
\end{tabular}

prevent one coronary event in the lower three quartiles would be about 140, suggesting the case for aggressive lifestyle intervention in these subjects before any consideration is given to the introduction of drug therapy. Economic constraints (see later) wield an ever-growing influence on clinical decision-making.

Another way to arrive at a decision on which patients should receive lipid-lowering drug therapy is to compare the benefits of treating hyperlipidemia in asymptomatic at-risk men with the results of other risk factor intervention studies. Two such studies have been compared elsewhere [13]: 4S, a trial of cholesterol lowering in patients with established CHD [14] and the MRC trial [15], a pri- mary prevention study of the effects of treating hypertension on the risk of stroke. Analysed in this way (table 4) approximately three times as many WOSCOPS patients had to be treated to save one CHD death or nonfatal MI, compared to patients with established CHD who were enrolled in 4S. Table 4 also shows that 2-4 times more hypertensives required to be treated to save one stroke in the MRC Trial, than patients with high cholesterol to save an MI in WOSCOPS.

At first glance these results suggest that the absolute benefits of treatment in WOSCOPS are less than have been shown for patients with established CHD, but that they compare favorably with the treatment of mild-to- 
moderate hypertension. Further analysis of event rates experienced by the WOSCOPS placebo group, as noted earlier, however, has shown that within a cohort of moderately hypercholesterolemic middle-aged men there exists a continuum of risk from low $(<5 \%$ coronary event rate over 5 years) to high ( $20 \%$ event rate over 5 years). Low-risk WOSCOPS subjects have isolated hypercholesterolemia and a coronary event rate similar to that recorded for stroke in the MRC Trial. High-risk WOSCOPS subjects are older men with overt or silent atherosclerotic disease whose coronary event rates approach those recorded in $4 \mathrm{~S}$. The number of low-risk and high-risk WOSCOPS subjects needed to be treated to save one major coronary event may then be estimated by calculating absolute risk reduction for different levels of baseline risk, assuming a comparable relative risk reduction of $30 \%$.

How useful is our comparison of WOSCOPS with the results of the MRC Trial? While matching of these two studies permits direct comparison of primary prevention interventions in men of the same age, based on data available in the main results paper of each trial, the following reservations should be noted. The two studies were conducted about 10 years apart. The study populations, key risk factors and end points were different. Perhaps most importantly, the entry criteria for the MRC Trial defined mild hypertension as diastolic BP $90-109 \mathrm{~mm} \mathrm{Hg}$ after 3 visits, during a run-in period of only 2 weeks [15]. Accepted definitions and classifications of hypertension have changed since the MRC Trial: some patients randomised to active treatment or placebo would not now be regarded as hypertensive, while others in this BP range would be said to have moderate hypertension, according to current guidelines [16]. It is likely that fewer hypertensive patients would need to be treated for 5 years to prevent one stroke if treatment was restricted to those in whom BP elevation was sustained over a more prolonged period of pre-treatment observation.

A subsequent analysis of placebo group patients in the MRC Trial [17] confirms that the slope of the relation between BP and risk is steeper when based on measurements made after 6 months of follow-up on placebo. The 5-year stroke risk for men and women aged 35-64 years whose systolic BP remained $160 \mathrm{~mm} \mathrm{Hg}$ or greater after 6 months was twice that of patients whose systolic BP was $160 \mathrm{~mm} \mathrm{Hg}$ upon entry to the trial (in many of whom BP spontaneously fell thereafter). However, the magnitude of any uncertainty created by this difference in treatment threshold is not sufficient to invalidate the comparisons we have drawn between WOSCOPS and the MRC Trial it remains the case that fewer hyperlipidemic patients must be treated to prevent one MI than hypertensives of similar age to prevent one stroke. It is certainly possible to increase the number of strokes prevented by treatment of hypertension, but in order to do so older patients must be targetted. Given that proportionate benefit in the stroke trials is comparable at around $40 \%$, it follows that absolute benefit is greatest among patients whose absolute risk of stroke is greatest (i.e. older patients) at the start of treatment [17].

In conclusion, analysis of subgroup data from WOSCOPS permits identification of men whose 5-year risk of a major coronary event is $10 \%$ or greater, sufficiently high to justify lipid-lowering drug therapy for primary prevention. The absolute benefits of treating such men are less than for men with established CHD but greater than the benefits of treating mild to moderate hypertension in men of similar age to reduce the risk of stroke. It is of course cheaper to treat a hypertensive with bendrofluazide than to prescribe pravastatin for hyperlipidemia, although more expensive drugs are frequently used in hypertension, and it is likely that competitive pressures will force a reduction in the price of lipid-lowering therapy in future. Thus, in light of the widespread acceptance of the value of treating hypertension, we feel it difficult to justify not offering lipid-lowering pharmacotherapy to atrisk middle-aged men with hypercholesterolemia. The results of WOSCOPS, in which lipid-lowering therapy produced statistically significant reductions in CHD death and nonfatal MI, all cardiovascular death and, for the first time, all cause mortality, clearly supports the use of a statin in such patients.

\section{Summary: Who should be treated?}

On the basis of WOSCOPS and the European Joint Guidelines, primary CHD prevention should focus on

a <55-year-old hypercholesterolemic men with either

- A smoking habit

- Diabetes

- A minor ECG abnormality

- Pre-existing vascular disease or

- Multiples of the above

b >55-year-old hypercholesterolemic men with any of the following: - Hypertension

- Diabetes

- A family history of CHD

- A low HDL cholesterol $(<1.1 \mathrm{~m} M ;<40 \mathrm{mg} / \mathrm{dl})$

- A smoking habit

- Pre-existing vascular disease

- A minor ECG abnormality

- Multiples of the above 


\section{Note:}

1 The above strategy applies to individuals with modest hypercholesterolemia $(<7.8 \mathrm{mM} ;<300 \mathrm{mg} / \mathrm{dl})$ and does not cover lipid abnormalities of genetic origin.

2 A minor ECG abnormality is defined as Minnesota Codes 4-2, 4-3 or 5-2, 5-3. This effectively is equivalent to ST depression $\leq 1 \mathrm{~mm}$ with a horizontal or downward sloping ST segment, or T wave inversion $<5 \mathrm{~mm}$. It should be noted, however, that in WOSCOPS which was a primary prevention study, individuals with more significant ST-T changes were excluded from the study. Thus, none of the participants had a left bundle branch block on entry or ST depression $>1 \mathrm{~mm}$ or T wave inversion $>5 \mathrm{~mm}$. It is to be expected that such findings would also constitute an even more significant risk factor.

3 Women were not examined in WOSCOPS.

\section{B What Is the Treatment Strategy?}

Both the Lipid Research Clinics Coronary Primary Prevention Trial [3, 4] and the Helsinki Heart Study [5] showed that lipid-lowering strategies were effective in reducing the risk of ischemic heart disease. However, the drugs used in these projects (cholestyramine and gemfibrozil) differ substantially in their effects on the lipoprotein profile and not surprisingly there was enthusiastic interest at that time in identifying those elements in the plasma lipid spectrum which have the greatest bearing on coronary risk and whose modification produces the best cardioprotective outlook. It transpired that both LDL and HDL levels in the plasma influenced risk, a reduction in the former or a rise in the latter being protective. The drugs employed in these trials, however, in contrast to the WOSCOPS project, were relatively ineffectual or disagreeable to patients and so the changes which they managed to induce in plasma lipids and lipoproteins were, in general, modest. The West of Scotland Study is therefore the first primary prevention trial with adequate statistical power to examine the relationship between plasma lipid levels and their influence on CHD events.

The West of Scotland Study plasma lipid analysis, which is the subject of a recent publication [18], addressed three fundamental questions:

(a) How do baseline lipids impact on the treatment benefits of statin therapy?

(b) Is there an association between LDL cholesterol reduction and event reduction?

(c) Does LDL reduction alone account for all of the cardioprotection offered by pravastatin?

Each of these issues is addressed in detail below.

\section{(a) How Do Baseline Lipids Impact on the Treatment}

Benefits of Statin Therapy?

In the West of Scotland Study, plasma lipid and lipoprotein concentrations (triglyceride, cholesterol, VLDL, LDL and HDL cholesterol) were measured according to the Lipid Research Clinics Protocol [19]. Measurements were made twice during screening, and individuals were included in the study if they had an LDL $\geq 4.0 \mathrm{mmol} / 1(155$ $\mathrm{mg} / \mathrm{dl})$ on both occasions and $\geq 4.5 \mathrm{mmol} / \mathrm{l}(174 \mathrm{mg} / \mathrm{dl})$ on one. If LDL exceeded $6.0 \mathrm{mmol} / 1(232 \mathrm{mg} / \mathrm{dl})$ at both screening visits, the subject was excluded. Men were randomized to receive placebo or pravastatin $40 \mathrm{mg} /$ day, and subsequent visits were conducted every 3 months. Fasting lipid profiles were obtained at 6-month intervals during the follow-up period. In the present analysis, baseline plasma lipid levels were taken as the mean of the values observed at the two screening visits. As noted in the publication of baseline characteristics [12], there was no significant difference in any lipid variables between these visits.

The endpoint used in this review is all-cardiac events, defined as the occurrence of fatal MI, other cardiovascular death, nonfatal MI, coronary artery bypass graft (CABG) or percutaneous transluminal coronary angioplasty (PTCA) as a first event. This provided approximately 50\% more events than the primary endpoint and hence enhanced power to detect associations and differences. CABG and PTCA as separate endpoints showed similar risk reduction on pravastatin to the primary endpoint.

All 6,595 randomized patients were used to calculate quintiles of mean baseline LDL cholesterol, HDL cholesterol, and plasma triglyceride. The Kaplan-Meier 5-year risk of an all-cardiac event was then determined separately for each quintile of the placebo and pravastatin groups. Baseline lipids as continuous variables were related to risk of an all-cardiac event in the two groups separately using Cox regression [20] both univariately and then multivariately with other baseline covariates [as described in ref. 12] to test their independence as predictors. The covariates employed in the adjustment were age, smoking, blood pressure, ECG abnormality, self-reported angina, self-reported hypertension, diabetes, family history of premature death from CHD, and nitrate use.

Baseline LDL cholesterol was a weak predictor of risk in both treatment groups largely because of the artificially narrow band of LDL cholesterol dictated by the entry criteria of the trial. Individuals in the top quintile of the placebo group experienced a rate for the all-cardiac endpoint of $12 \%$ per 5 years compared with $9 \%$ in the bottom quintile (fig. 3a). The proportionate reduction in risk of an event was similar across all quintiles in patients taking 


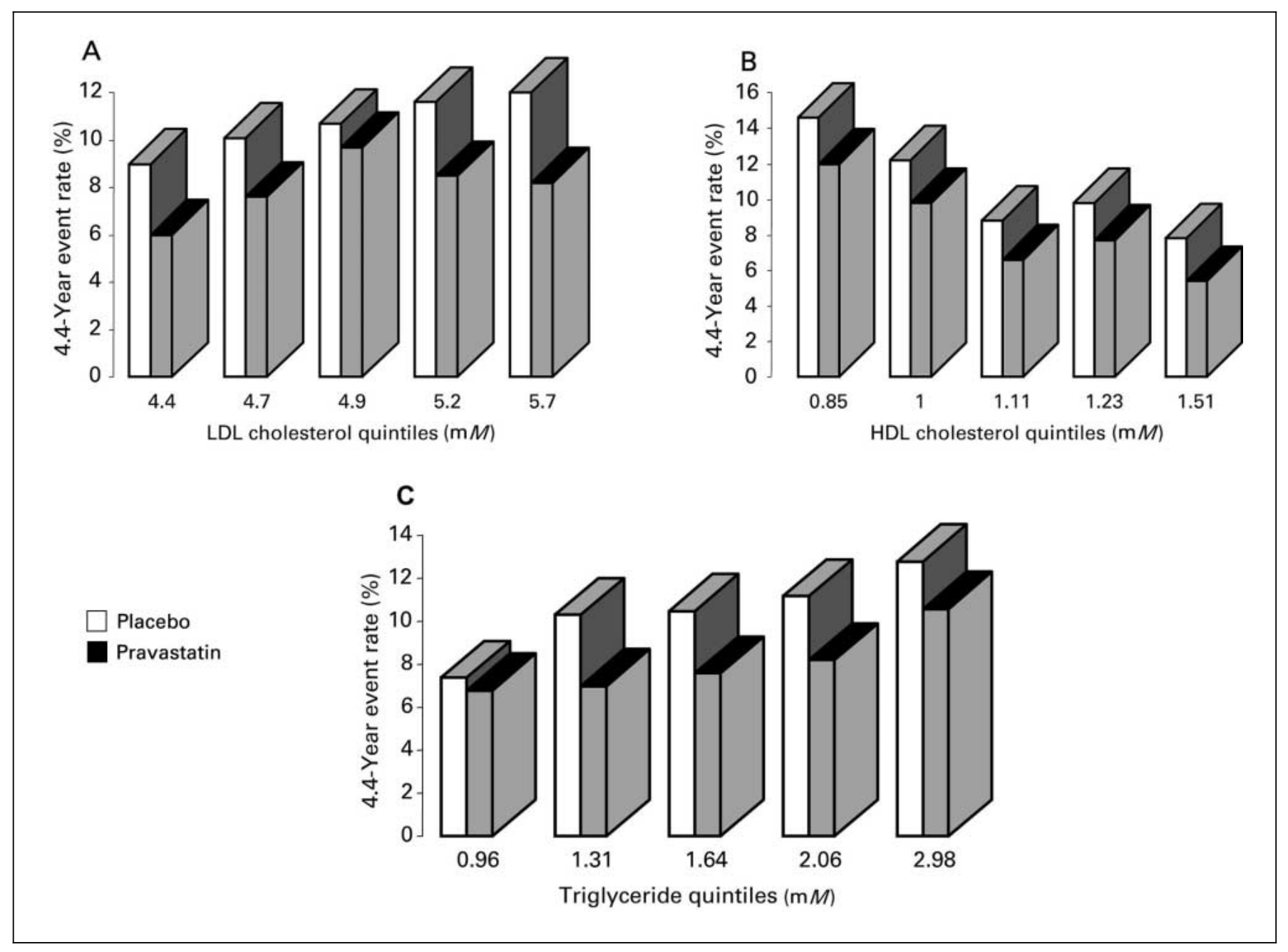

Fig. 3. Baseline lipids and treatment benefits from pravastatin. Subjects in the placebo and pravastatin-treated groups in WOSCOPS were divided into quintiles according to their mean baseline LDL cholesterol (a), HDL cholesterol (b) or plasma triglyceride (c). Kaplan-Meier 5-year estimated event rates were determined for each quintile. Solid columns = Placebo; hatched columns = pravastatin. To convert $\mathrm{mmol} / \mathrm{l}$ cholesterol to $\mathrm{mg} / \mathrm{dl}$, multiply by 38.7 ; to convert $\mathrm{mmol} / \mathrm{l}$ triglyceride to $\mathrm{mg} / \mathrm{dl}$, multiply by 88.5 .

pravastatin. Baseline HDL cholesterol exhibited a clear negative association with event rate (fig. $3 b$ ) and was a major predictor of CHD risk in both treatment arms of the study. Again, the relative risk reduction was similar for all quintiles of this lipid fraction. The plasma triglyceride level at baseline was positively related to the risk of CHD (fig. 3c). Patients receiving placebo who had a baseline triglyceride level of $\geq 2.3 \mathrm{mmol} / \mathrm{l}(204 \mathrm{mg} / \mathrm{dl})$ had almost twice the event rate of patients with an initial triglyceride level less than $1.2 \mathrm{mmol} / \mathrm{l}(106 \mathrm{mg} / \mathrm{dl})$ despite having similar baseline LDL levels (mean of $5.0 \mathrm{mmol} / \mathrm{l}$ ) $(194 \mathrm{mg} / \mathrm{dl})$ in all quintiles of plasma triglyceride). On the basis of univariate analysis, the starting triglyceride value was a highly significant predictor of risk in both groups. In line with previous observations [21], inclusion of baseline HDL in multivariate models led to a loss of significance of baseline plasma triglyceride as a predictor.

The above results permit us to conclude that pravastatin therapy confers the same benefit to hypercholesterolemic (plasma cholesterol $=6.5-7.8 \mathrm{mmol} / 1 ; 250-300 \mathrm{mg}$ / dl) individuals, independently of baseline LDL, HDL and triglyceride. By the same token, and as reported previously [1], the proportionate gain from the therapy is uninfluenced by age, smoking status, or the presence of symp- 
Fig. 4. LDL reduction versus event reduction in WOSCOPS. Subjects receiving pravastatin were divided into quintiles of percentage decrease from baseline in LDL as a result of treatment. Kaplan-Meier 4.4-year risks of an event were calculated for each quintile. Similar calculations relating event reduction to absolute LDL cholesterol reduction gave comparable results since baseline LDL cholesterol values for each quintile were essentially the same.

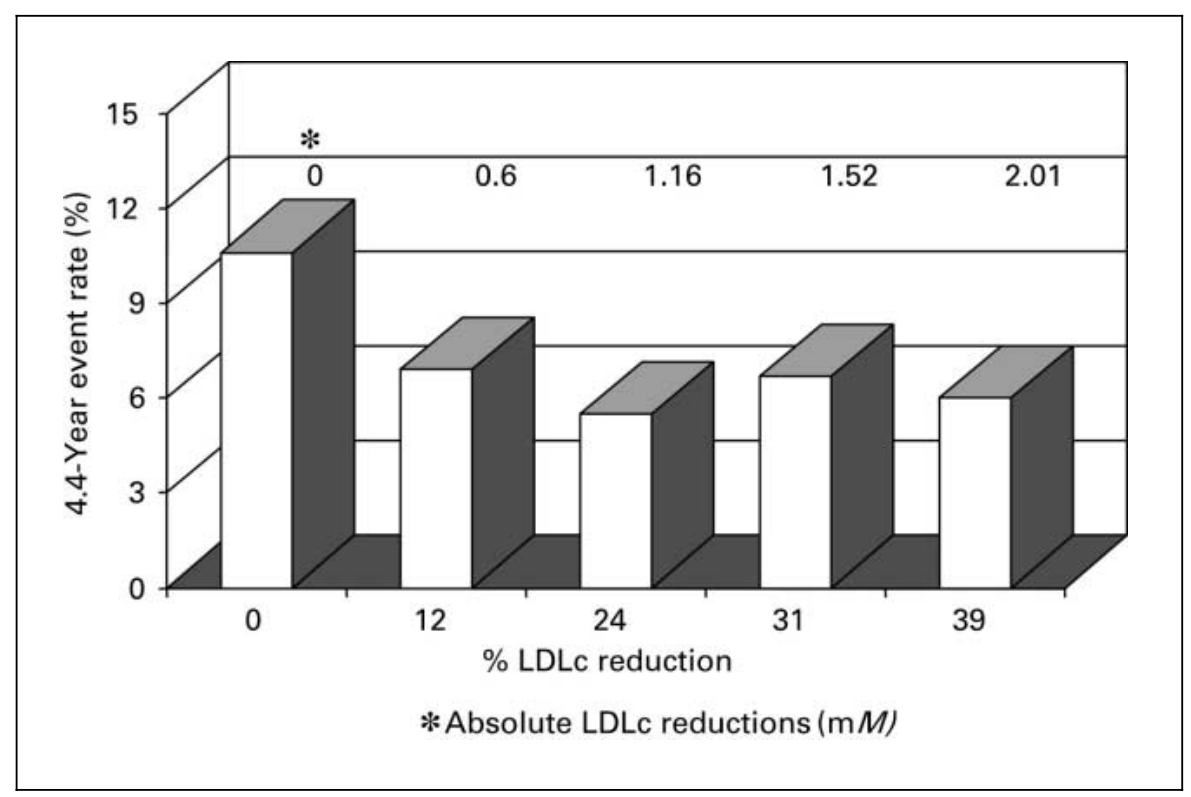

toms of CHD. So, within the constraints of the lipid inclusion criteria in WOSCOPS, all subjects were equally helped by the drug.

\section{(b) Is There an Association between LDL Cholesterol} Reduction and Event Reduction?

There is heated ongoing debate over the value of aggressive total and LDL cholesterol reduction in the prevention of coronary heart disease. The protagonists assert that increasing benefit will accrue from greater lipid lowering, implying that there is a linear association between plasma cholesterol values and coronary risk. But, the Multiple Risk Factor Intervention Trial [22], the largest observational study of its kind, and other similar large-scale prospective projects make it clear that populations experience a curvilinear rise in coronary events with increasing plasma cholesterol. By inference, then, cholesterol-lowering strategies would most effectively reduce events in individuals with more severe hypercholesterolemia and would become attenuated in their capacity to do so as individual plasma cholesterol values fell. In other words, a patient will receive greater cardioprotection from the first, say, $25 \%$ cholesterol reduction than from efforts to lower cholesterol by $25 \%$ more. This principle of diminishing returns was tested in the present WOSCOPS analysis, using the following approach. Firstly, the percentage reduction of LDL cholesterol from baseline was calculated for each individual in the pravastatin-treated group. To provide the most accurate measure of plasma lipid concentration during follow-up, on-treatment lipid values were calculated at the mean of all lipid measurements made after randomization until the patient had an event or reached the end of the study. If a lipid value was missing at a visit but study medication had been issued at the previous visit (3 months earlier), the most recent measurement that had been preceded by a medication issue was carried forward. If before the visit no such on-treatment measurement existed, then the baseline value was imputed. Baseline value was also imputed if no medication had been issued at the previous visit and the present lipid level was missing. Secondly, the Kaplan-Meier 4.4-year risk of a cardiac event (as specified in section (i) above) was determined for each quintile of percent LDL reduction. Each quintile was then compared with the whole placebo group in a Cox model. In this analysis, the first 6 months of follow-up in both groups were excluded, since no on-treatment lipid values were available for patients who had an event before this time had elapsed. The difference between treatments was assessed with and without adjustment for potential baseline covariate imbalance and expressed as risk ratios relative to placebo with $95 \%$ confidence limits. To determine if quintiles differed from each other with respect to risk of an all-cardiac event, quintiles 1 through 4 were compared in Cox multivariate models with quintile 5 (highest percent change in LDL).

The percentage fall in LDL cholesterol during treatment varied (fig. 4) even in patients who complied with the treatment regimen (i.e. they attended and had study 
medication) issued on at least $75 \%$ of the scheduled visits). When the pravastatin group was divided into quintiles of percentage LDL reduction (based on measured plus imputed values), it was observed that the mean change varied from 0 to $-39 \%$ (fig. 4). We expected that a decrease in LDL would be the major determinant of risk reduction and that a strong, graded association would be present between these two variables. However, figure 4 illustrates that in quintiles 2 through 5 there was no obvious correlation between percent LDL reduction and event rate. When adjustment was made for baseline covariates, the relative risk in quintile 4 vs. 5 was $1.39(\mathrm{p}=$ $0.14)$; in quintile 3 vs. $5,1.09(\mathrm{p}=0.72)$; in quintile 2 vs. 5 , $1.43(\mathrm{p}=0.11)$; and in quintile 1 vs. 5, $2.24(\mathrm{p}=0.001)$. Thus, quintiles 2 through 4 did not differ significantly from quintile 5 in terms of cardiovascular risk reduction achieved; and individuals in quintile 1, who achieved no LDL reduction, had the same CHD risk as the entire placebo group over the five years of the study. So, if LDL levels were not reduced by treatment, there was no cardiovascular gain, but a fall of $24 \%$ was sufficient to produce maximum benefit. Whatever additional gain (if any) came from driving LDL lower did not translate into further measurable cardioprotection.

\section{(c) Does LDL Reduction Alone Account for All of the Cardioprotection Offered by Pravastatin?}

The inexplicably early appearance of benefit in WOSCOPS which resulted within six months in visible divergence of the cumulative event curves in the placebo and pravastatin treated cohorts raised the possibility that change in lesion size and, by inference, LDL cholesterol reduction alone might not fully explain the actions of the drug in reducing coronary events. Two independent approaches were made to examine this issue using: (i) overlap analysis, (ii) comparison with Framingham.

(i) Overlap Analysis. There was considerable overlap in the distribution of mean LDL cholesterol in the placebo and pravastatin treated cohorts, 1,120 individuals on placebo and 1,071 on pravastatin therapy having an LDL cholesterol between 3.62 and $4.65 \mathrm{mmol} / 1$ (140-180 mg/ dl). To be included in the overlap analysis, patients had to be more than $75 \%$ compliant and should not have had an event in the first six months of follow-up. Risk of cardiac event (fatal MI or other cardiovascular death, nonfatal MI, CABG or PTCA) was then compared between the groups. Event rates for the two subgroups differed markedly. Pravastatin treatment was associated with a $36 \%$ $(95 \%$ confidence interval $=56-9 \%)$ lower risk $(\mathrm{p}=0.014$; fig. 5), a finding that did not appear to be due to an imbal-

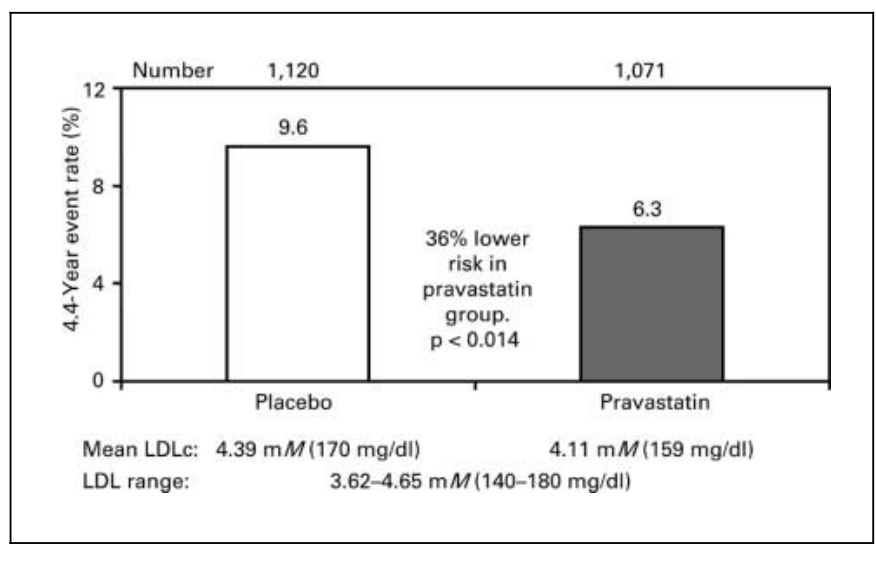

Fig. 5. Overlap analysis in WOSCOPS. The frequency distribution for LDL cholesterol in the placebo and pravastatin-treated subjects overlap substantially. Subjects in each cohort with LDL cholesterol between 3.62 and $4.65 \mathrm{mmol} / \mathrm{l}(140$ and $180 \mathrm{mg} / \mathrm{dl})$ were compared. Despite having the same LDL cholesterol values as their placebo treated counterparts, pravastatin treated individuals exhibited significantly fewer cardiovascular events (definite or suspect CHD death or nonfatal MI, other cardiovascular death or revascularisation) over the 4.4 years of observation.

ance in baseline risk factors or to differences in on-treatment LDL (or on-treatment plasma triglyceride, VLDL cholesterol or HDL cholesterol). Similar differences (data not shown) were obtained on examination of a narrower $3.88-4.39 \mathrm{mmol} / 1(150-170 \mathrm{mg} / \mathrm{dl})$ overlap, a region where the non-treatment LDL values were virtually equal in the two groups ( 4.23 vs. $4.15 \mathrm{mmol} / 1$ (164 vs. $161 \mathrm{mg} /$ dl) for placebo- and pravastatin-treated subjects, respectively).

(ii) Comparison with Framingham. The equation published by the Framingham investigators [23-25] permits calculation of the risk of a CHD event based on gender, age, plasma cholesterol, HDL cholesterol, smoking habit, systolic blood pressure and presence of diabetes. This model was employed as a further approach to test the hypothesis that the event reduction seen in patients taking pravastatin could not be explained entirely by changes in the measured plasma lipid levels. To maintain compatibility with the Framingham coronary event definition, the endpoint in this instance was taken as definite nonfatal MI or CHD death plus revascularization (PTCA and CABG). Again, patients were omitted from the analysis if they had experienced a coronary event, had cancer, or had undergone angiography within 6 months of randomization; and in order to maintain compatibility with the Framingham cohort, men with baseline angina, as determined by nitrate use or positive Rose questionnaire, were 


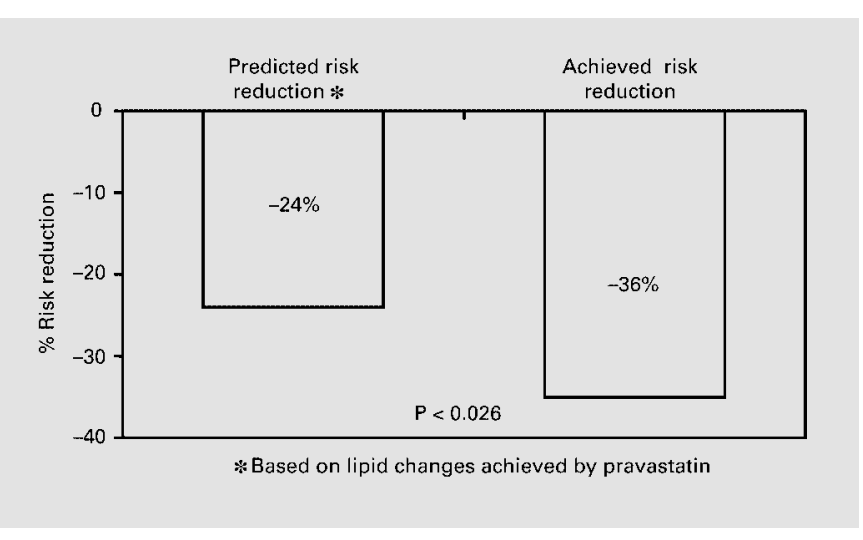

Fig. 6. WOSCOPS and the Framingham Study. The predicted cardiovascular risk over 4.4 years for each pravastatin treated WOSCOPS participant was derived from the Framingham risk equation $(23,24,25)$ and compared to the observed risk reduction achieved in WOSCOPS for the combined endpoint of fatal and nonfatal MI, CABG and PTCA. Only patients who fell into the range of plasma lipid levels and blood pressure readings seen in Framingham were included in the analysis. The pravastatin treated cohort expressed significantly fewer events (a $35 \%$ risk reduction) than predicted from Framingham (a 24\% risk reduction).

excluded. Inclusion necessitated that patients fall into the plasma cholesterol $(4.13-7.23 \mathrm{mmol} / \mathrm{l} ; 160-280 \mathrm{mg} / \mathrm{dl})$ and blood pressure (diastolic, 70-105 $\mathrm{mm} \mathrm{Hg}$; systolic $110-170 \mathrm{~mm} \mathrm{Hg}$ ) ranges which characterised the Framingham population from which the risk equation was derived. They also had to comply with the treatment regimen and to have at least one on-treatment lipid measurement available. Prediction of risk was again made from the point at which on-treatment lipid levels were available (6 months after post-randomization) over the remaining period of the trial ( 4.4 years, since the mean total length of follow-up was 4.9 years). Predicted event rates were derived for each subject using his mean (with imputation when necessary) on-treatment level for plasma cholesterol and HDL cholesterol. The number of predicted and observed events were compared in the pravastatin group. The predicted fall in risk of CHD determined from the Framingham equation was $24 \%$ while, in actual fact a $35 \%$ reduction was observed in the WOSCOPS trial (fig. 6). This difference was highly significant $(\mathrm{p}<0.026)$.

There was a number of possible explanations for the findings of the overlap analysis and the Framingham comparison. First, patients who experience a sudden reduction in LDL cholesterol may benefit from, at least for a time, a lower risk than those who naturally have an LDL at that concentration. Second, in addition to lowering LDL, pravastatin has been shown to promote the removal of triglyceride-rich remnant particles from the bloodstream [26]. These lipoprotein species have been linked to the progression of atherosclerotic lesions and their clearance, which is known to occur through receptormediated pathways [27], may lead to stabilization of plaques whose rupture might otherwise have given rise to clinical events. Third, pravastatin may, through pathways not involving lipid lowering [28], beneficially affect atherosclerosis (e.g. by decreasing a tendency for thrombosis or by inhibiting the inflammatory response which characterises atheromatous plaques). The latter two possibilities could account for the relatively early benefit seen during pravastatin therapy in WOSCOPS. It is noteworthy that other lipid-lowering therapies $[3,4,29]$ that work by stimulating receptor-mediated catabolism of LDL (i.e. bile acid sequestrant resins and surgical biliary diversion) did not show an early treatment effect, although they did provide long-term risk reduction. Possibly this difference in response arises because these therapeutic approaches enhance VLDL production in the liver and do not have the same impact on the plasma concentration of remnants of triglyceride-rich lipoproteins as does pravastatin.

The results described here are derived from post hoc analysis and therefore must be viewed cautiously. Nevertheless, they indicate that the benefit seen with pravastatin treatment, although obviously linked to a decrease in LDL, cannot be explained by this alone.

\section{Summary: What is the treatment strategy?}

Detailed analysis of the lipid data in WOSCOPS shows that

- Baseline levels of triglyceride and LDL and HDL cholesterol were predictive of CHD risk

- All subjects gain the same relative benefit from pravastatin, independently of their baseline lipid profile and within the framework of the WOSCOPS lipid inclusion criteria

- There is no cardiovascular gain from pravastatin therapy unless LDL cholesterol levels are reduced, but a fall of about $25 \%$ is sufficient to produce maximum benefit in modestly hypercholesterolemic men

- LDL reduction alone does not appear to account entirely for the benefit of pravastatin therapy

\section{How Much Will It Cost?}

So far we have considered whom to treat and how aggressively to approach their problem. The third key issue which we must explore is the question of how much the prevention strategy will cost. With this objective in mind, an economic model of cardiovascular disease pre- 
Table 5. Treatment costs per life-year gained based on coronary risk stratification in the West of Scotland Study cohort

\begin{tabular}{lllc}
\hline $\begin{array}{l}\text { Average annual } \\
\text { risk of a } \\
\text { CHD event, \% }\end{array}$ & \begin{tabular}{l} 
Cost $(£)$ per life-year gained \\
\cline { 2 - 4 }
\end{tabular} & (a) undiscounted & $\begin{array}{l}\text { \% of WOS popu- } \\
\text { lation requiring } \\
\text { treatment }\end{array}$ \\
\hline 1.6 & $8,121($ KD 3,621.966) & $20,375($ KD 9,087.250) & 100 \\
2.0 & $5,601($ KD 2,498.046) & $13,995($ KD 6,241.770) & 23 \\
3.0 & $3,900($ KD 1,739.400) & $9,682($ KD 4,318.172) & 8 \\
\hline
\end{tabular}

vention was created [30] based on the premise that the first cardiovascular event (be it the appearance of angina, a nonfatal myocardial infarction or the need for coronary revascularisation) is seen by the individual and by society as a whole as an irreversible transition from health to sickness, and therefore worthy of prevention.

To estimate the effects of pravastatin on this process, the WOSCOPS database was interrogated to identify the various events which constitute the transition. Pravastatin treatment was compared to the effect of the placebo, each intervention being added to basal dietary advice. The process allowed for a progression of events occurring in the same individual and was conducted at monthly intervals for 60 months (i.e. the mean duration of the trial). The net consequence of not using pravastatin was the cumulative difference in transitions (fig. 7). The number needed to treat to prevent one transition differed in its definition from the usual approach in that it identified those who require to start treatment (rather than those who continued on treatment) to avoid a transition.

Calculation of the number of life years gained by those on treatment made use of the unique Scottish Record Linkage System [31] which is a database containing details on more than 460,000 cardiovascular events which were managed in Scotland between 1981 and 1995. This database allowed forward projection of what would have happened to individuals who would have succumbed to an event had it not been for pravastatin therapy. The cost of any transition was based on the average direct 1996 price of managing each type of event, averaged from information supplied by 200 hospitals in Scotland. All costs were discounted at $6 \%$ to satisfy Treasury recommendations. The cost of the pravastatin treatment itself (£ 1.66 (KD 0.740) per 40-mg tablet) was calculated from the number of people receiving one tablet daily; and incidental costs of monitoring the subject's progress was estimated on the basis of current practice guidelines (GP visit, liver function tests, lipoprotein analysis, etc.).

Finally, in order to assess the effect of varying risk in different patient subgroups on the price of intervention, individuals were collected into groupings based on the

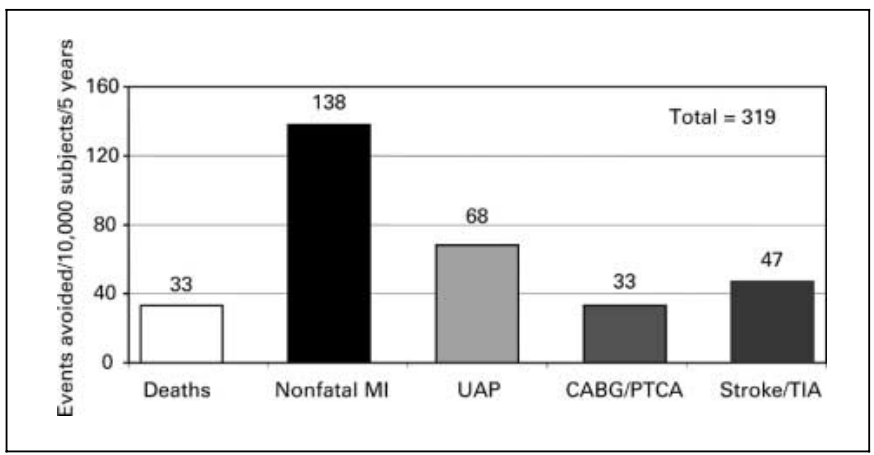

Fig. 7. Vascular events avoided in WOSCOPS through the use of pravastatin therapy.

risk factors [12] of age, diagnosis of hypertension, diastolic blood pressure, baseline HDL cholesterol, smoking habit, ECG abnormality, family history of CHD, widowhood, positive Rose questionnaire, previous vascular disease, diabetes and the use of glyceryl trinitrate.

Pravastatin treatment of the hypercholesterolemic asymptomatic WOSCOPS men avoided 319 transitions in 10,000 men treated over 5 years (fig. 7). That is, to prevent one transition, 31.4 men would require to receive treatment for this time. There are several ways of measuring the implications of avoiding these transitions. For example, eliminating the events would have saved 2,017 hospital bed days or extended life in the 10,000 individuals by 2,460 years. The cost of the drug for the 10,000 men would have been $£ 23,340,984$ (KD 10,410,078), offset by $£ 529,214$ (KD 236,029.44) in savings from treatment of the prevented diseases. So, overall, discounting at $6 \%$ would have led to a cost of $£ 20,375$ (KD 9,087.25) per life year gained. This assumes that all shades of risk in the WOSCOPS trial were accommodated. If one limits treatment, in line with the European Joint Guidelines [11], to those individuals with a $20 \%$ risk of an event over the next 10 years (i.e. approximately $2 \%$ per annum), then the numbers requiring treatment to avoid an event would fall to 22.5 and the cost of extending life by 1 year would fall (discounted at 6\% pa) to $£ 13,995$ (KD 6,241.77). 
Finally, if a more rigorous approach were taken, and only individuals with a $3 \%$ risk of an event were selected, the discounted cost of each life year gained would be $£ 9,680$ (KD 4,317.28). Selection of this target would mean treating $8 \%$ of the 35 - to 69 -year-old Scottish population (table 5). So, although widespread use of pravastatin for primary prevention may seem [31] like an unjustified extravagance imposed on an already strained healthcare system, the WOSCOPS results provide good evidence that this intervention, if judiciously applied, is economically sound.

\section{Summary: How much will it cost?}

From the WOSCOPS results

- Starting 10,000 men on pravastatin would help 319 of them avoid a transition from health to cardiovascular disease

- If all WOSCOPS men were treated, the cost of adding one year to life would be $£ 20,375$ (KD 9,087.25) (discounted at 6\%)

- If, according to the Joint European Guidelines, those with an annual risk of $2 \%$ were selected for treatment, the cost per life year gained would be $£ 13,995$ (KD 6,241.77)

- If only those with an annual risk of $3 \%$ were selected for treatment, the cost per life year gained would be $£ 9,680$ (KD 4,317.28); eight percent of the 35- to 69-year-old Scottish population would require to be treated

\section{References}

1 Shepherd J, Cobbe SM, Ford I, Isles CG, Lorimer AR, Macfarlane PW, McKillop JH, Packard CJ: Prevention of coronary heart disease with pravastatin in men with hypercholesterolemia. N Engl J Med 1995;333:13011307.

2 A co-operative trial in the primary prevention of ischaemic heart disease using clofibrate: Report from the Committee of Principal Investigators. Br Heart J 1978;40:1069-1118.

3 The Lipid Research Clinics Coronary Primary Prevention Trial Results. I. Reduction in incidence of coronary heart disease. JAMA 1984; 251:351-364

4 The Lipid Research Clinics Coronary Primary Prevention Trial Results. II. The relationship of reduction in incidence of coronary heart disease to cholesterol lowering. JAMA 1984;251: 365-374.

5 Frick MH, Elo O, Haapa K, et al: Helsinki Heart Study: Primary prevention trial with gemfibrozil in middle-aged men with dyslipidemia: Safety of treatment, changes in risk factors, and incidence of coronary heart disease. $\mathrm{N}$ Engl J Med 1987;317:1237-1245.

6 Oliver MF: Might treatment of hypercholesterolemia increase non-cardiac mortality? Lancet 1991;337:1529-1531.

7 Hulley SB, Walsh JMB, Newman TB: Health policy on blood cholesterol: Time to change directions. Circulation 1992;86:1026-1029.

8 Davey Smith G, Pekkanen J: Should there be a moratorium on the use of cholesterol lowering drugs? BMJ 1992;304:431-434.

9 Rose G, McCartney P, Reid DD: Self administration of a questionnaire on chest pain: Intermittent claudication. Br J Prevent Soc Med 1977;31:42-48.

10 The West of Scotland Coronary Study Group: Compliance and adverse event withdrawal: Their impact on the West of Scotland Coronary Prevention Study. Eur Heart J 1997;18:17181724.

11 Wood D, de Backer G, Faergeman O, et al, on behalf of the Task Force: Prevention of coronary heart disease in clinical practice. Recommendations of the Second Joint Task Force of European and other Societies on Coronary Prevention. Atherosclerosis 1998;140:199-270.
12 The West of Scotland Coronary Prevention Study Group: Baseline risk factors and their association with outcome in the West of Scotland Coronary Prevention Study. Am J Cardiol 1997;79:756-762.

13 The West of Scotland Coronary Prevention Study Group: West of Scotland Coronary Prevention Study: Identification with high risk groups and comparison with other cardiovascular intervention trials. Lancet 1996;348: 1339-1342.

14 The Scandinavian Simvastatin Survival Study Group: Randomised trial of cholesterol lowering in 4444 patients with coronary heart disease: The Scandinavian Simvastatin Survival Study (4S). Lancet 1994;344:1383-1389.

15 Medical Research Council Working Party: MRC Trial of treatment of mild hypertension: Principal results. BMJ 1985;291:97-104.

16 Sever P, Beevers G, Bulpitt C, Lever A, Ramsay L, Reid J, Swales J: Management guidelines in essential hypertension: Report of the second working party of the British Hypertension Society. BMJ 1993;306:983-987.

17 Lever AF, Ramsay LE: Treatment of hypertension in the elderly. J Hypertens 1995;13:571579.

18 West of Scotland Coronary Prevention Study Group: Influence of pravastatin and plasma lipids on clinical events in the West of Scotland Coronary Prevention Study. Circulation, in press.

19 Lipid Research Clinics Manual of Laboratory Operations: Washington, Government Printing Office, 1975, DHEW publ No. (NIH) 85268.

20 Collett D: Modelling Survival Data in Medical Research. London, Chapman Hall, 1994.

21 Austin MA: Plasma triglyceride and coronary heart disease. Arterioscler Thromb 1990;11:114.

22 Martin MJ, Hulley SB, Browner WS, Keeler LH, Wentworth D: Serum cholesterol, blood pressure and mortality: Implications from a cohort of 361,662 men. Lancet 1986;ii:933936 .
23 Anderson KM, Odell PM, Wilson PWF, Kannel WB: Cardiovascular disease risk profiles. Am Heart J 1990;121:293-298.

24 Anderson KM, Wilson PWF, Odell PM, Kannel WB: An updated coronary risk profile. Circulation 1991;83:357-363.

25 Abbott RD, McGee D: The Framingham Study, Section 37: The probability of developing certain cardiovascular diseases in eight years at specified values of some characteristics. NIH Publ No 87-2284, 1987.

26 Knopp RH, Illingworth DR, Stern EA, Ginsberg HN, Broyles EE, Behounek BD: Effect of pravastatin in the treatment of patients with type III hyperlipoproteinemia. Am J Ther 1996;3:755-762.

27 Gaw A, Packard CJ, Murray EF, Lindsay GM, Griffin BA, Caslake MJ, Vallance BD, Lorimer AR, Shepherd J: Effects of simvastatin on apoB metabolism and LDL subfraction distribution. Arterioscler Thromb Vasc Biol 1993;13:170189.

28 Shepherd J: Pleiotrophism among the statins. Br J Cardiol 1997;4(suppl 1):S28-S31.

29 Buchwald H, Varco RL, Matts JP, Long JM, Fitch LL, Campbell GS, Pearce MB, Yellin AE, Edmiston WA, Smink RD Jr, et al, the POSCH Group: Effect of partial ileal bypass surgery on mortality and morbidity from coronary heart disease in patient with hypercholesterolemia: Report of the program on the surgical control of the hyperlipidemias (POSCH). N Engl J Med 1990;323:946-955.

30 Caro J, Klittich W, McGuire A, Ford I, Norrie J, Pettitt D, McMurray J, Shepherd J: The West of Scotland Coronary Prevention Study: Economic Benefit Analysis of Primary Prevention with Pravastatin. Br Med J 1997;315: 1577-1582.

31 Kendrick S, Clarke J: The Scottish record linkage system. Health Bull (Edin) 1993;51:72-79.

32 Pharoah PDP, Hollingworth W: Cost effectiveness of lowering cholesterol concentration with statins in patients with and without pre-existing coronary heart disease: Life table method applied to health authority population. Br Med J 1996;312:1443-1448. 